\title{
Linking Emergency Medical Service Data to Death Records for Opioid Mortality Surveillance
}

\author{
Joseph R. Tatar, Jennifer Broad \\ Wisconsin Department of Health Services, Madison, Wisconsin, United States \\ Objective
}

To identify the correlates of opioids as an underlying cause of death by linking coroner/medical examiner vital death records with emergency medical service (EMS) ambulance run data. By combining death data to EMS ambulance runs, the goal was to determine characteristics of the emergency response - particularly for opioid overdose events - that may connect to increased mortality.

\section{Introduction}

Opioid abuse has increased exponentially in recent years throughout the United States, leading to an increase in the incidence of emergency response activities, hospitalization, and mortality related to opioid overdose. As a result, states that have been hit particularly hard during this period — such as Wisconsin - have allocated considerable resources to addressing this crisis via enhanced public health surveillance and outreach, procurement and administration of medical countermeasures, prescription drug monitoring programs, targeted preventive and acute treatment, first responder and hospital staff training, cross-agency collaboration, and Incident Management System activities. Central to these efforts is the identification of the primary drivers of opioid overdose and death to improve the precision and efficacy of targeted public health interventions to address the opioid crisis. The present study sought to accomplish this end by syncing rich data sources at the point of emergency response (EMS ambulance runs) to ultimate mortality outcomes (vital death records).

\section{Methods}

In the State of Wisconsin, data systems supporting the surveillance of EMS ambulance runs and coroner/medical examiner death records are both maintained under the Department of Health Services, enhancing the ability of public health researchers to connect these records using matched identifiers. Two years of EMS ambulance run data (2016-2017) were matched to three years of vital death records (2016-2018) that listed opioids as a contributing cause of death. Ambulance runs and death records for individuals aged 10 years or younger were removed from the data prior to matching and were not included in the final analytic set. Records between these two systems were matched using patient first and last name, gender, date of birth, and zip code. Ambulance runs for a suspected opioid overdose were identified by mining text fields from EMS primary and secondary impressions as well as incident narrative details that identified an opioid as a likely cause of the event. Ambulance runs resulting in Narcan/naloxone administration were also identified as opioid-related overdose. Coroner/medical examiner death records that identified opioids as a contributing cause were classified as an opioid-related death. Analyses examining correlates of deaths with opioids as a contributing cause focused on patient demographics, Narcan/naloxone administration rates and dosage, date and time of the ambulance run, lag between EMS response and time of opioid-related death, physical location and urbanicity of the incident, and the type of response by EMS personnel (i.e. treated and transported, treated and released, no treatment, patient refusal, DOA).

\section{Results}

From 2016-2017, there were over 800,000 emergency ambulance runs among those aged 11 years and older. Opioid overdose ambulance runs accounted for $1.1 \%(9,761)$ of those runs. There were over 100,000 deaths in Wisconsin and 1.7\% $(1,797)$ were related to opioids (i.e. opioids were a contributing cause). Linking resulted in 268 people with opioid overdose ambulance runs who had an opioid-related death. Of these, $34 \%$ died at the scene of the ambulance run, $12 \%$ died later that day, $16 \%$ died within a week of the ambulance run, and 37\% died after a week. While all of these deaths had a contributing cause of opioids, $97 \%$ also had an underlying cause of death of drug overdose. Comparing those who died to those who didn't die, those who died were more likely to be male, younger, and had the event occur on a Saturday. Additionally, while there were no differences in the likelihood of Narcan/naloxone receipt by opioid-related death, individuals who died were more likely to have received multiple Narcan/naloxone doses during the ambulance run than those who did not. Of those who died at the scene, the majority (32\%) were aged 30 to 39 years. Of those who died later, the majority (32\%) were aged 20 to 29 years. Also, for those who died at the scene,

SDS Annual Conference Proceedings 2019. This is an Open Access article distributed under the terms of the Creative Commons AttributionNoncommercial 4.0 Unported License (http://creativecommons.org/licenses/by-nc/3.0/), permitting all non-commercial use, distribution, and reproduction in any medium, provided the original work is properly cited. 
the majority of the events occurred from eight pm to midnight while for those who died later, the majority of events occurred from four to eight $\mathrm{pm}$.

\section{Conclusions}

The majority of linked deaths to opioid ambulance runs were due to an underlying cause of drug overdose with opioids as a contributing cause. This demonstrates that the impressions of the EMS personnel were correct. The fact that so many of those who died did so at the scene highlights the continued need for community naloxone distribution. Additionally, there appear to be characteristic differences between those who died, those who died at the scene, and those who didn't die. The results from this study highlight the benefits of connecting multiple sources of data to facilitate the identification of emergency health care drivers of opioid-related death, but there is still work to be done. Future analyses from this project will seek to link the existing data to hospitalization and post-discharge care records to capture a more complete picture of opioid-related deaths across the entire patient lifecycle. This future work will serve to fill key gaps in the surveillance process, particularly for instances opioid overdose and death where EMS was not called into service.

\section{Acknowledgement}

We would like to thank the Office of Health Informatics and the Office of Preparedness and Emergency Health Care, especially the EMS Section for their support of this project. Funding for this study comes from the Enhanced State Opioid Overdose Surveillance grant, generously provided by the Centers for Disease Control and Prevention. 